$\begin{array}{cc}\text { Programa de Pós-Graduação em Engenharia de Produção - PPGEP } & \\ \text { Laboratório de Qualidade de Vida - LaQVida } & \\ \text { Universidade Tecnológica Federal do Paraná - UTFPR } & \text { REVISTA BRASILEIRA DE } \\ \text { Ponta Grossa - PR - Brasil } & \text { QUALIDADE DE VIDA }\end{array}$

, n. 04 , out./dez. 2015 , p. $210-215$

DOI: $10.3895 /$ rbqv.v7n4.3157

\title{
Relação entre o IMC dos pais e dos filhos
}

\section{Relationship between BMI of parents and children}

\author{
William Cordeiro de Souza \\ Universidade do Contestado - UnC - Canoinhas - Santa Catarina - Brasil \\ professor williamsouza@yahoo.com.br \\ Marcos Tadeu Grzelczak \\ Universidade do Contestado - UnC - Porto União - Santa Catarina - Brasil \\ promarcostadeu@hotmail.com \\ Luis Paulo Gomes Mascarenhas \\ Universidade Estadual do Centro-Oeste - Unicentro - Irati - Paraná - Brasil \\ masca58@hotmail.com
}

\section{RESUMO}

OBJETIVO: Verificar a relação entre o Índice de Massa Corporal (IMC) dos pais e dos filhos.

MÉTODOS: A amostra intencional foi constituída por 36 adolescentes, com média de idade de $16,2 \pm 1,0$ anos, e seus respectivos pais, pai com média de idade 43,6 \pm 3 anos e mãe com idade média 38,3 $\pm 6,9$ anos. Para a obtenção do IMC dos adolescentes foi coletado os dados de peso e estatura, sendo calculado a partir da fórmula: $\mathrm{IMC}=$ Peso $(\mathrm{kg}) /$ altura $(\mathrm{m})^{2}$. O IMC dos pais foi determinado pelo próprio autorrelato do peso corporal e da estatura, através de questionário preenchido por eles. Para as classificações do IMC foi utilizou-se os pontos de corte sugeridos pela World Health Organization (2004). No tratamento estatístico foi utilizado o software Med Calc, onde se calculou a estatística descritiva (Média e Desvio Padrão - DP). Foi realizado o teste de normalidade de Spearman para determinação do grau de correlação entre o IMC dos pais com o dos filhos, adotando-se como limiar de significância $\mathrm{p}<0,05$.

RESULTADOS: Foi verificada relação significativa entre o IMC dos pais versus IMC dos filhos $(\mathrm{r}=0,43 ; \mathrm{p}=0,01)$; e entre o IMC das Mães versus $\mathrm{IMC}$ dos filhos $(\mathrm{r}=0,46 ; \mathrm{p}=0,004)$.

CONCLUSÕES: Os resultados demonstraram que existem relações positiva e moderada entre o IMC dos pais sobre o IMC dos filhos independente de sua classificação.

PALAVRAS-CHAVE: Composição corporal. Sobrepeso/obesidade. Ambiente familiar.

\section{ABSTRACT}

OBJECTIVE: Check the relationship between the BMI of parents and children.

METHODS: The intentional sample consisted of 36 adolescents with a mean age of $16.2 \pm 1.0$ years, and their parents, father with a mean age $43.6 \pm 3$ year-old mother with a mean age $38.3 \pm$ 6.9 years. To obtain the BMI of adolescents were collected data on weight and height, is calculated 
from the formula: $\mathrm{BMI}=$ weight $(\mathrm{kg}) /$ height $(\mathrm{m})^{2}$. The BMI of the parents was determined by the very self-reported weight and height, in the questionnaire completed by them. For BMI classifications were used the cutoff points suggested by World Health Organization (2004). In the statistical analysis we used the Med Calc software, where it calculated the descriptive statistics (mean and SD). Was performed Spearman normality test to determine the degree of correlation between parental BMI with the children, adopting as significance threshold of $p<0.05$.

RESULTS: Significant relationship was found between BMI of the parents of the children versus BMI ( $r=0.43 ; p=0.01)$; and between BMI Mother versus BMI of children ( $r=0.46 ; p=0.004)$.

CONCLUSIONS: The results showed that there is positive and moderate relationship between parental BMI on the BMI of children regardless of their classification.

KEYWORDS: Body composition. Overweight/obesity. Family environment.

\section{Introdução}

Nos últimos 40 anos, houve no Brasil um aumento significativo de, pelo menos 5 vezes, na prevalência de sobrepeso e obesidade entre crianças e adolescentes em ambos os sexos (MIRANDA et al., 2015; PUDLA; GONZALÉZ-CHICA; VASCONCELOS, 2015).

Esse problema de saúde pública está aumentando gradativamente em todas as faixas etárias, especialmente na população infantil e adolescente, onde suas consequências colaboram para o aumento da morbimortalidade e de outras doenças não transmissíveis, sendo as mais comuns diabetes e hipertensão arterial (BERNARDO et al., 2010; PAULA et al., 2014).

De acordo com Frignani et al. (2015), o maior período de incidência da obesidade é a transição entre a adolescência e as etapas precoces da vida adulta. Vários fatores são determinantes para o aumento da obesidade, entretanto estudos têm demonstrado que a obesidade dos pais pode influenciar diretamente no peso dos filhos (BERNARDO et al., 2010; MASCARENHAS et al., 2013).

Segundo Ramos e Barros Filho (2003), a herança genética acrescida das influências ambientais são fatores determinantes para o risco de ocorrência da obesidade. Dalcastagné et al. (2008) acrescentam que essa influência pode ser exercida por fatores biológicos, psicológicos, socioeconômicos e sociocomportamentais que contribuem para o desenvolvimento da obesidade.

Mascarenhas et al. (2013) inferem que o ambiente familiar, presença de apenas um dos pais, o número de irmãos, nível sociocultural são variáveis que também apresentam relação com a obesidade.

Diante do exposto, o presente estudo tem por objetivo verificar a relação entre o Índice de Massa Corporal (IMC) dos pais e dos filhos.

\section{Métodos}

A coleta de dados ocorreu em uma instituição de curso técnico (jovem aprendiz) da cidade de Canoinhas - SC. O presente estudo teve a aprovação do coordenador do estabelecimento para que a pesquisa fosse realizada. Após a aprovação do coordenador, foi entregue aos alunos um termo de consentimento livre e esclarecido, explicando os procedimentos a serem utilizados, e um questionário a ser preenchido pelos pais, o qual solicitava informações sobre idade, peso e estatura. Foram excluídos os jovens com qualquer comprometimento físico que impedisse a avaliação antropométrica e aqueles que fizessem uso de medicamentos que pudessem influenciar no crescimento. Vale ressaltar que a presente pesquisa seguiu os princípios éticos de respeito à autonomia das pessoas, apontada pela Resolução $n^{\circ}$ 196, de 10 de outubro de 1996, do Conselho Nacional de Saúde. 
A instituição de ensino tem 61 alunos matriculados nos cursos de elétrica e mecânica. $\mathrm{O}$ questionário e o termo de consentimento foram entregues a todos os alunos. Apenas 36 adolescentes retornaram com as devidas informações solicitadas.

Para a obtenção do IMC dos adolescentes foi coletada a estatura por meio de fita métrica fixa em uma parede plana, com os adolescentes em posição ereta, descalços, com os pés unidos e paralelos (FERNANDES FILHO, 2003). A mensuração do peso corporal foi obtida através de balança digital portátil da marca da marca Filizola, com capacidade máxima de $150 \mathrm{~kg}$. O IMC foi calculado a partir da fórmula: $\mathrm{IMC}=$ Peso $(\mathrm{kg}) /$ altura $(\mathrm{m})^{2}$.

O IMC dos pais foi determinado pelo próprio autorrelato do peso corporal e da estatura, através do questionário preenchido por eles. $O$ autorrelato apresenta correlação de $0,90 \mathrm{com}$ a avaliação própria do indivíduo (SPENCER et al., 2002). Para as classificações do IMC dos pais e dos filhos foi utilizou-se os pontos de corte sugeridos pela World Health Organization (2004) conforme Quadro 1:

Quadro 1 - Classificação do IMC

\begin{tabular}{|c|c|}
\hline IMC & Classificações \\
\hline Menor do que 18,5 & Abaixo do Peso Normal \\
\hline $18,5-24.99$ & Peso Normal \\
\hline $25,00-29,99$ & Excesso de Peso \\
\hline $30,00-34,99$ & Obesidade Grau I \\
\hline $35,00-39,99$ & Obesidade Grau II \\
\hline Maior ou Igual a 40,00 & Obesidade Grau III \\
\hline
\end{tabular}

Fonte: World Health Organization (2004).

No tratamento estatístico foi utilizado o software Med Calc, onde se calculou a estatística descritiva: Média, Desvio Padrão (DP) e Percentual (\%). Foi realizado o teste de normalidade de Spearman para determinação do grau de correlação entre o IMC dos pais com o dos filhos, adotando-se como limiar de significância $\mathrm{p}<0,05$.

\section{Resultados}

A amostra foi constituída por 36 adolescentes do sexo masculino, com média de idade de $16,2 \pm 1,0$ anos, e seus respectivos pais, pai com média de idade $43,6 \pm 3$ anos e mãe com idade média $38,3 \pm 6,9$ anos.

A Tabela 1 apresenta os dados: peso, estatura e IMC para caracterização da amostra.

Tabela 1 - Caracterização da amostra

\begin{tabular}{cccc}
\hline Variáveis & Pai $(\mathbf{n}=\mathbf{3 6})$ & Mãe $(\mathbf{n}=36)$ & Filhos $(\mathbf{n}=\mathbf{3 6})$ \\
\hline Peso $(\mathbf{k g})$ & $82,2 \pm 10,6$ & $66,5 \pm 10,0$ & $64,9 \pm 13,5$ \\
Estatura $(\mathbf{m})$ & $1,8 \pm 0,1$ & $1,7 \pm 0,1$ & $1,7 \pm 0,1$ \\
IMC $\left(\mathbf{k g} / \mathbf{m}^{2}\right)$ & $26,1 \pm 2,8$ & $24,1 \pm 3,5$ & $21,2 \pm 3,1$ \\
\hline \multicolumn{4}{c}{ Fonte: Autoria própria $(2015)}$.
\end{tabular}

O Gráfico 1 apresenta a relação entre o IMC dos pais versus IMC dos filhos, onde foi encontrada uma correlação significativa $(r=0,43 ; p=0,01)$. 


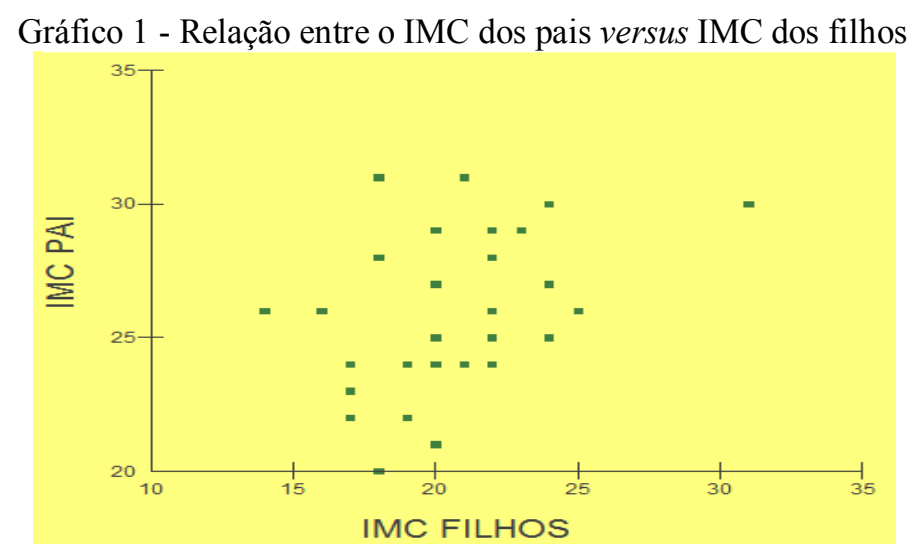

Fonte: Autoria própria (2015).

Já o Gráfico 2 apresenta a relação entre o IMC das Mães versus IMC dos filhos, onde também foi encontrada uma correlação significativa $(r=0,46 ; p=0,004)$.

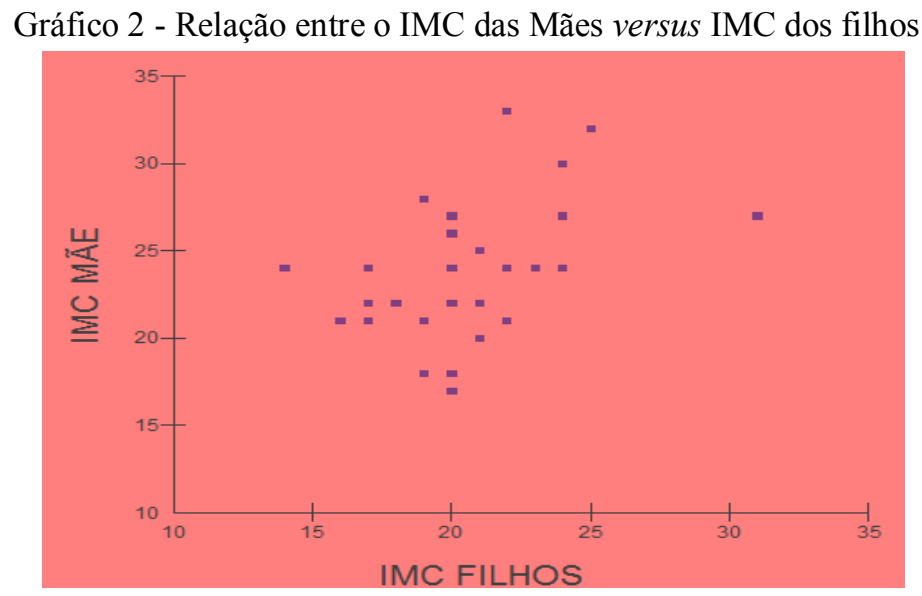

Fonte: Autoria própria (2015).

No Gráfico 3 são apresentadas a distribuição de frequência do IMC, segundo a classificação da Organização Mundial da Saúde.

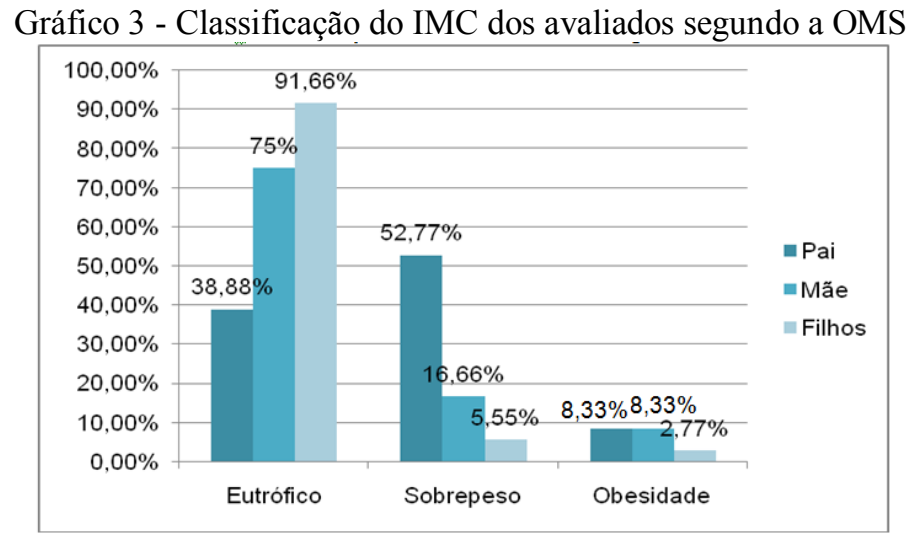

Fonte: Autoria própria (2015).

\section{Discussão}

A presente pesquisa teve como objetivo verificar a relação entre o IMC dos pais e o IMC dos filhos. Os dados demonstraram que existe relação entre o IMC dos pais versus IMC dos filhos, o mesmo aconteceu entre o IMC das Mães versus IMC dos filhos. Foi verificado que $61,1 \%$ dos 
pais avaliados apresentaram sobrepeso e obesidade. Já em relação às mães $25,0 \%$ apresentaram seu peso acima do normal.

Ramos e Barros Filhos (2003) apresentam estimativas que a criança com pais obesos tem $80 \%$ de chance de se tornar obesa, enquanto que a proporção diminui para $40 \%$ quando apenas o pai ou a mãe é obeso. No presente estudo percebeu-se que os avaliados têm uma grande chance de se tornar um adulto obeso, pois houve um número considerável de pais acima do recomendado. Vale ressaltar que apenas $8,32 \%$ dos adolescentes estão acima do peso.

Corroborando com os dados do presente estudo, Petroski e Pelegrini (2009), com o intuito de verificar a associação entre o estilo de vida dos pais e a composição corporal de seus filhos, observaram que o estilo de vida dos pais estava associado à composição corporal dos filhos.

Em estudo realizado por Coelho e Pires (2014), evidenciou-se que há uma grande predominância do ambiente familiar no ganho de peso das crianças. Tendo sido verificado que ocorre uma maior incidência de excesso de peso em crianças com famílias pouco funcionais.

Zambon e Tumelero (2007 destacam que existe uma grande relação entre o IMC do pai com o do filho. O IMC da mãe também apresenta relação, porém não tão significativa quanto ao valor apresentado pelo do pai. Dados esses que contrapõem ao presente estudo onde foram encontradas relações semelhantes pai $(\mathrm{r}=0,43)$ e mãe $(\mathrm{r}=0,46)$, sendo que a relação do IMC das mães foi maior quando relacionado com o IMC dos pais, apesar do grupo de mães avaliadas serem classificadas como eutróficas.

Mascarenhas et al. (2013) investigaram a influência do IMC dos pais sobre o IMC dos seus filhos e verificaram que o risco relativo (RR) do adolescente com pai com sobrepeso/obesidade de apresentar sobrepeso/obesidade é de 1,97 vezes maior. Este valor sobe para 2,28 quando a mãe apresenta sobrepeso/obesidade e para 2,65 quando ambos os pais têm sobrepeso/obesidade. Este estudo mostrou que sobrepeso/obesidade de ambos os pais aumenta o RR de excesso de peso das adolescentes femininas, enquanto que a obesidade materna é um fator de risco tanto dos adolescentes masculinos como femininos.

Vale ressaltar que o presente estudo não investigou hábitos alimentares, nível de atividade física, gasto calórico e outras variáveis que poderiam interferir nos resultados. Sendo assim, sugerese que, para próximas pesquisas, controlar essas variáveis, assim serão construídos mais parâmetros de diferentes situações e verificado o que elas causam e contribuem contra a obesidade.

\section{Considerações finais}

Ao finalizar o estudo foi verificado que existem relações positivas e moderadas entre o IMC dos pais sobre o IMC dos filhos independente de sua classificação.

\section{Referências}

BERNARDO, C. O.; FERNANDES, P. S.; CAMPOS, R. M. M. B.; ADAMI, F.;

VASCONCELOS, F. A. G. Associação entre o índice de massa corporal de pais e de escolares de 7 a 14 anos de Florianópolis, SC, Brasil. Revista Brasileira de Saúde Materno Infantil, v. 10, n. 2, p. 183-190, 2010. Crossef

COELHO, H. M.; PIRES, A. P. Relações familiares e comportamento alimentar. Psicologia: Teoria e Pesquisa, v. 30, n. 1, p. 45-52, 2014. Crossef

DALCASTAGNÉ, D.; RANUCCI, J. M. A.; NASCIMENTO, M. A.; LIBERALI, R. A influência dos pais no estilo de vida dos filhos e sua relação com a obesidade infantil. Revista Brasileira de Obesidade, Nutrição e Emagrecimento, v. 2, n. 7, p. 44-52, 2008. 
FERNANDES FILHO, J. A prática da avaliação física: testes, medidas e avaliação física em escolares, atletas e academias de ginástica. 2. ed. Rio de Janeiro: Shape, 2003.

FRIGNANI, R. R.; PASSOS, M. A. Z.; FERRARI, G. L. M.; NISKIER, S. R.; FISBERG, M. CINTRA, I. P. Reference curves of the body fat index in adolescents and their association with anthropometric variables. Jornal de Pediatria, v. 91, n. 3, p. 248-255, 2015. Crossef

MASCARENHAS, L. P. G. MODESTO. M. J.; AMER, N. M.; BOGUSZEWSKI, M. C. S.; LACERDA FILHO, L.; PRATI, F. S. Influência do excesso de peso dos pais em relação ao sobrepeso e obesidade dos filhos. Revista Pensar a Prática, v. 16, n. 2, p. 519-532, 2013. Crossef

MIRANDA, J. M. Q.; PALMEIRA, M. V.; POLITO, L. F. T.; BRANDÃO, M. R. F.; BOCALINI, D. S.; FIGUEIRA JUNIOR, A. J.; PONCIANO, K.; WICHI, R. B. Prevalência de sobrepeso e obesidade infantil em instituições de ensino: públicas vs. privadas. Revista Brasileira de Medicina do Esporte, v. 21, n. 2, p. 104-107, 2015. Crossef

PAULA, F. A. R.; LAMBOGLIA, C. M. G. F.; SILVA, V. T. B. L.; MONTEIRO, M. S.; MOREIRA, A. P.; PINHIRO, M. H. N. P.; SILVA, C. A. B. Prevalência de sobrepeso e obesidade em escolares da rede pública e particular da cidade de Fortaleza. Revista Brasileira de Promoção da Saúde, v. 27, n. 4, p. 455-461, 2014. Crossef

PETROSKI, E. L.; PELEGRINI, A. Associação entre o estilo de vida dos pais e a composição corporal dos filhos adolescentes. Revista Paulista de Pediatria, n. 27, n. 1, p. 48-52, 2009.

PUDLA, K. J.; GONZALÉZ-CHICA, D. A.; VASCONCELOS, F. A. G. Effect of breastfeeding on obesity of schoolchildren: influence of maternal education. Revista Paulista de Pediatria, 2015 (no prelo). doi: 10.1016/j.rpped.2015.01.004. Crossef

RAMOS, A. M. P. P; BARROS FILHO, A. A. Prevalência da obesidade em adolescentes de Bragança Paulista e sua relação com a obesidade dos pais. Arquivos Brasileiros de Endocrinologia e Metabologia, n. 47, n. 6, p. 663-668, 2003.

SPENCER, E. A.; APPLEBY, P. N.; DAVEY, G. K.; KEY, T. J. Validity of self-reported height and weight in 4808 EPIC-Oxford participants. Public Health Nutrition, v. 5, n. 4, p. 561-565, 2002. Crossef

WORLD HEALTH ORGANIZATION (WHO). Obesity: preventing and managing the global epidemic. 2004. Disponível em: $<$ http://apps.who.int/bmi/index.jsp?introPage=intro 3.html $>$. Acesso em: 06 mar. 2015.

ZAMBON, T. B.; TUMERELO, S. Relação corporal através do IMC entre adolescentes e seus pais. EFDeportes.com, Revista Digital, Buenos Aires, v. 11, n. 105, p. 1, 2007.

\section{Conflitos de interesse}

Não há nenhum potencial conflito de interesse entre os autores desse trabalho. 